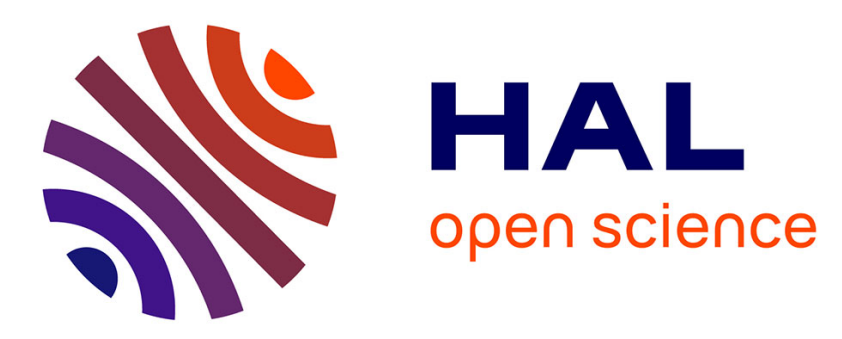

\title{
Piezocomposite transducer design and performance for high resolution ultrasound imaging transducers
}

Fidèle Hanse Wampo, Roger Pierre Lemanle Sanga, Pierre Maréchal, Guy-Edgar Ntamack

\section{- To cite this version:}

Fidèle Hanse Wampo, Roger Pierre Lemanle Sanga, Pierre Maréchal, Guy-Edgar Ntamack. Piezocomposite transducer design and performance for high resolution ultrasound imaging transducers. International Journal of Computational Materials Science and Engineering, 2019, 08 (03), pp.1950013. 10.1142/S2047684119500131 . hal-02483625

\section{HAL Id: hal-02483625 \\ https://hal.science/hal-02483625}

Submitted on 21 Jul 2021

HAL is a multi-disciplinary open access archive for the deposit and dissemination of scientific research documents, whether they are published or not. The documents may come from teaching and research institutions in France or abroad, or from public or private research centers.
L'archive ouverte pluridisciplinaire HAL, est destinée au dépôt et à la diffusion de documents scientifiques de niveau recherche, publiés ou non, émanant des établissements d'enseignement et de recherche français ou étrangers, des laboratoires publics ou privés. 


\title{
Piezocomposite transducer design and performance for high resolution ultrasound imaging transducers
}

\author{
F.L. Hanse Wampo ${ }^{1}$, R.P. Lemanle Sanga ${ }^{1}$, P. Maréchal ${ }^{2}$, G.E. Ntamack ${ }^{1}$, \\ ${ }^{1}$ Groupe de Mécanique des Matériaux et de l'Acoustique (GMMA), Université de Ngaoundéré, Cameroun. \\ ${ }^{2}$ Laboratoire Ondes et Milieux Complexes (LOMC), UMR 6294 CNRS, Université du Havre, France. \\ Corresponding author: pierre.marechal@univ-lehavre.fr
}

\begin{abstract}
Piezocomposite design for dedicated ultrasonic imaging applications requires precise homogenization models for predicting the electromechanical characteristics of the new material. Thus several homogenization models have been developed. As part of this work, we applied several analytical homogenization models for piezocomposite of 2-2 and 1-3 connectivities. To validate these analytical models, a comparative study was made between various models and experimental measurements. As a result, these homogenized electromechanical properties are effectively used for the calculation and comparison of electroacoustic response for typical transducers aimed at ultrasound imaging applications. An optimal design of transducer aimed at ultrasound imaging applications is proposed as a dedicated imaging performance index, elaborated through a trade-off between sensitivity and bandwidth.
\end{abstract}

Keywords : piezoelectric materials; connectivity; homogenization; effective properties; ultrasound imaging transducers.

\section{Introduction}

In the past decades, the application fields of ultrasonic waves for detection and characterization have developed considerably. Piezoelectric materials are mostly used as active material for ultrasound applications and both their compositions and fabrication methods have known strong developments, especially in submarine detection systems and medical imaging [1,2]. Although significant progress has been made on these materials, new piezoelectric composites structures were born of the increased need for sensitivity and applications. Among them, 2-2, 0-3 and 1-3 composite connectivities were shown as promising configurations [1]. In general, their use tends to grow in the mobile and embedded electronics. Applications of piezoelectric materials require improvement of their characteristics. The electromechanical behavior of the composite media essentially involves multiscale methods [3-6]. However, because of their high manufacturing cost, and high prices are generally used in numerical modeling for the study of their micro and macroscopic mechanical behavior. Thus several homogenization models have been developed for calculating the actual 
coefficients. In this work, we make a comparison of some analytical models applied to piezocomposites having 2-2 and 1-3 connectivities. On the basis of connectivity concept, we write the constitutive equations of piezoelectricity. Some analytical homogenization models are first described. Then these different models of homogenization are applied in some types of piezocomposite. To validate analytical models, a comparison between the analytical results and the experimental values is discussed. An application of such piezocomposite transducers to ultrasound medical imaging is studied. The electroacoustic performance of optimally designed piezocomposite transducers is compared for various damping configurations in a given electrical and front medium environments. The electroacoustic performances of transducers aimed at ultrasound imaging applications is proposed as a result of a dedicated performance index. Finally, the relevance of this index is demonstrated through the comparison of the transfer functions and electroacoustic responses of a basic and two optimum configurations, each one corresponding to the studied 2-2 and 1-3 connectivities.

\section{Homogenization models for piezoelectric composites}

\subsection{Connectivity}

To define how the phases are coupled therebetween, Newnham et al. [7] have introduced the concept of connectivity. The connectivity is the number of directions in which it is possible to cross from one side without leaving the cube considered phase this figure is from 0 to 3 . A composite is then classified according to the connectivity of each phase, the first digit is by convention that of the piezoelectric phase. For a two-phase composite, Figure 1 shows the various possible connectivities.
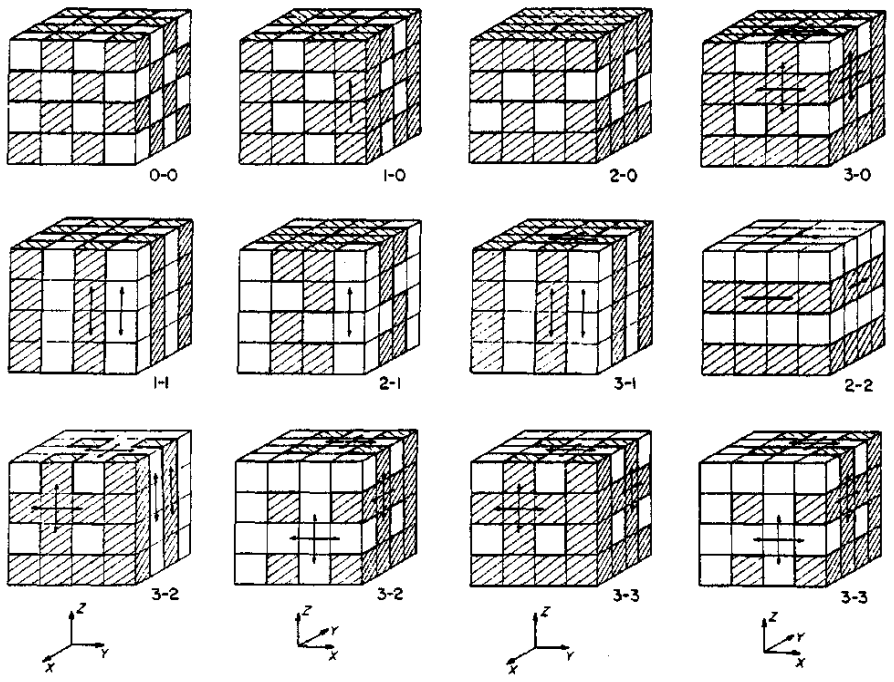

Figure 1: Various connectivities for a two-phase composite: phase 1 (clear) stands for the piezoelectric phase and phase 2 (shaded) stands for the polymer phase [7]. 


\subsection{Constitutive equations}

For an infinitesimal deformation, the components of strain tensor of order 2 are defined by the following equation [8]:

$$
S_{i j}=\frac{1}{2}\left(\frac{\partial u_{i}}{\partial x_{j}}+\frac{\partial u_{j}}{\partial x_{i}}\right)
$$

where $S_{i j}$ are the strain tensor components, the indexes $i$ and $j$ are in $\{1,2,3\}, u_{i}$ is a component of the displacement vector, and $x_{i}$ is the considered axis.

In a piezoelectric material, electrical and mechanical phenomena interact, resulting in electromechanical effects. Neglecting the pyroelectric effect, fundamentals of piezoelectricity can be written as a generalized Hooke's equation:

$$
\left\{\begin{array}{l}
T=C^{E} S-e^{t} E \\
D=e S+\varepsilon^{S} E
\end{array} \equiv\left[\begin{array}{l}
T \\
D
\end{array}\right]=\left[\begin{array}{cc}
c^{E} & -e^{t} \\
e & \varepsilon^{S}
\end{array}\right]\left[\begin{array}{l}
S \\
E
\end{array}\right] \equiv[T D]=[K][S E]\right.
$$

where $[T D]=[T, D]^{t}$ is the generalized stress vector including $T$ the stress components and $D$ the electrical displacement components ; $[S E]=[S, E]^{t}$ is the generalized strain vector including $S$ the strain components and $E$ the electrical field components; $\left\{c^{E}, e, \varepsilon^{S}\right\}$ are the components of the generalized stiffness matrix $[K]$ including $c^{E}$ the stiffness components at constant electrical field, $e$ the piezoelectric components, and $\varepsilon^{S}$ the dielectric components at constant strain.

\section{Some analytical homogenization models}

When considering the general problem, the homogenization can be considered as a linear combination of the elementary components properties. Basically, in linear elasticity, the upper and lower bounds of the homogenization models are the sum of the stiffness coefficients (Voigt) [9] and the addition of the reciprocals compliance coefficients of the constituting phases (Reuss) [10], respectively:

$$
\left\{\begin{array}{l}
c_{33}^{\text {Voigt }}=\sum_{i=1}^{N} v_{i} c_{33}^{i} \\
c_{33}^{\text {Reuss }}=\left(\sum_{i=1}^{N} v_{i}\left(c_{33}^{i}\right)^{-1}\right)^{-1}
\end{array}\right.
$$

\subsection{Voigt and Reuss models}

In the case where the number of considered phases is $N=2$, some simplifications occurs and the Voigt [9] and Reuss [10] models results in: 


$$
\left\{\begin{array} { l } 
{ c _ { 3 3 } ^ { \text { Voigt } } = v _ { p } c _ { 3 3 } ^ { p } + v _ { m } c _ { 3 3 } ^ { m } } \\
{ c _ { 3 3 } ^ { \text { Reuss } } = \frac { c _ { 3 3 } ^ { m } c _ { 3 3 } ^ { p } } { v _ { m } c _ { 3 3 } ^ { p } + v _ { p } c _ { 3 3 } ^ { m } } }
\end{array} \equiv \left\{\begin{array}{l}
c_{33}^{\text {Voigt }}=c_{33}^{m}+v_{p}\left(c_{33}^{p}-c_{33}^{m}\right) \\
c_{33}^{\text {Reuss }}=\frac{c_{33}^{m}}{1-v_{p} \frac{c_{33}^{p}-c_{33}^{m}}{c_{33}^{p}}}
\end{array}\right.\right.
$$

where $\left\{v_{p}, v_{m}\right\}$ are the volume fraction of the piezoelectric and matrix phases, respectively, and is reduced to a single variable in the case of a two phases composite, since $v_{m}=1-v_{p}$.

\subsection{Matrix homogenization model}

The matrix method for the homogenization of piezocomposite is an analytical method proposed for calculating equivalent composite coefficients. Hashimoto and Yamaguchi [11] applied this model in the case of the 2-2 composite; Levassort et al. [12] applied it to the 0-3 and 1-3 composites.

\subsubsection{Continuity assumption}

The continuity assumption led to suppose that there is no relative movement between the two phases. This leads to the conclusion (equation (5)) that one hand, the stress $T$ and the electric displacement $D$ are continuous in the directions perpendicular to the interface and secondly that the deformations $S$ and the electric field $E$ are continuous in the directions parallel to the interface.

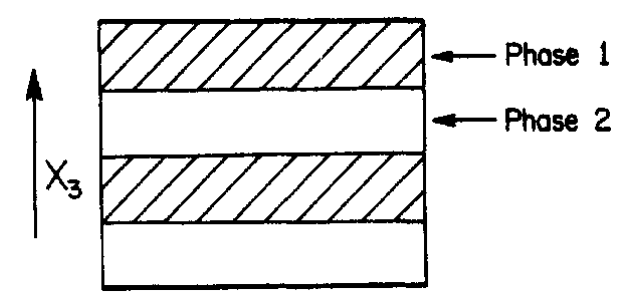

Figure 2: Basic piezocomposite cell for a 2-2 connectivity [7].

After applying this assumption 2-2 connectivity composite (Figure 2), the following relations are obtained continuity:

$$
\left(\begin{array}{l}
T_{1}^{e f f}=v_{p} T_{1}^{p}+v_{m} T_{1}^{m} \\
T_{2}^{\text {eff }}=v_{p} T_{2}^{p}+v_{m} T_{2}^{m} \\
S_{3}^{e f f}=v_{p} S_{3}^{p}+v_{m} S_{3}^{m} \\
S_{4}^{\text {eff }}=v_{p} S_{4}^{p}+v_{m} S_{4}^{m} \\
S_{5}^{\text {eff }}=v_{p} S_{5}^{p}+v_{m} S_{5}^{m} \\
T_{6}^{\text {eff }}=v_{p} T_{6}^{p}+v_{m} T_{6}^{m} \\
D_{1}^{\text {eff }}=v_{p} D_{1}^{p}+v_{m} D_{1}^{m} \\
D_{2}^{\text {eff }}=v_{p} D_{2}^{p}+v_{m} D_{2}^{m} \\
E_{3}^{\text {eff }}=v_{p} E_{3}^{p}+v_{m} E_{3}^{m}
\end{array}\right.
$$

and

$$
\left(\begin{array}{c}
S_{1}^{e f f}=S_{1}^{p}=S_{1}^{m} \\
S_{2}^{\text {eff }}=S_{2}^{p}=S_{2}^{m} \\
T_{3}^{\text {eff }}=T_{3}^{p}=T_{3}^{m} \\
T_{4}^{\text {eff }}=T_{4}^{p}=T_{4}^{m} \\
T_{5}^{\text {eff }}=T_{5}^{p}=T_{5}^{m} \\
S_{6}^{\text {eff }}=S_{6}^{p}=S_{6}^{m} \\
E_{1}^{\text {eff }}=E_{1}^{p}=E_{1}^{m} \\
E_{2}^{\text {eff }}=E_{2}^{p}=E_{2}^{m} \\
D_{3}^{\text {eff }}=D_{3}^{p}=D_{3}^{m}
\end{array}\right.
$$




\subsubsection{New expression of the constitutive law}

First, two matrix vectors $[H]$ and $[G]$ are defined, depending on discontinuity and continuity of parameters (equation (5)). Second, another matrix function $[W]$ that binds $[H]$ and $[G]$ is deduced:

$$
\left\{\begin{array}{l}
{[H]=\left[T_{1}, T_{2}, S_{3}, S_{4}, S_{5}, T_{6}, D_{1}, D_{2}, E_{3}\right]} \\
{[G]=\left[S_{1}, S_{2}, T_{3}, T_{4}, T_{5}, S_{6}, E_{1}, E_{2}, D_{3}\right]}
\end{array}\right.
$$

By introducing the $(9 \times 9)$ matrices $[P]$ and $[Q]$, we obtain:

$$
\left\{\begin{array}{l}
{[T D]=[Q][H]-[P][G]} \\
{[S E]=[Q][G]-[P][H]}
\end{array}\right.
$$

where $P_{i j}=\left\{\begin{aligned}-1 & \text { if } i=j=\{3,4,5,9\} \\ 0 & \text { else }\end{aligned}\right.$ and $Q_{i j}=\left\{\begin{aligned}+1 & \text { if } i=j=\{1,2,6,7,8\} . \\ 0 & \text { else }\end{aligned}\right.$.

By substituting $[H]=[W][G]$ in equation (7), and relating it with $[T D]=[K][S E]$ (equation (2)), we get:

$$
\left\{\begin{array}{l}
{[W]=[[K][P]+[Q]]^{-1}[[P]+[K][Q]]} \\
{[K]=[[Q][W]-[P]][[Q]-[P][W]]^{-1}}
\end{array}\right.
$$

The formalism described here is generic and can be easily transposed to other connectivities, as detailed by Levassort et al. [12] for the 0-3 connectivity, and Smith et al. [13] for the 1-3 connectivity.

\subsubsection{Piezocomposite electromechanical coefficients matrix}

The electromechanical behavior of the piezocomposite is given by $[T D]=[K][S E]$ (equation (2)). Moreover, following the continuity of relationship, $[G]$ is the same in the matrix, the fiber and the composite, while $[H]$ in the composite is the pondered average of those in the matrix and in the fiber. Thus we have:

$$
\left\{\begin{array}{l}
{[H]=v_{p}\left[H^{p}\right]+v_{m}\left[H^{m}\right]} \\
{[G]=\left[G^{p}\right]=\left[G^{m}\right]}
\end{array}\right.
$$

By substituting $[H]=[W][G]$ in equation (9) for both phases, we obtain:

$$
[H]=v_{p}\left[W^{p}\right]\left[G^{p}\right]+v_{m}\left[W^{m}\right]\left[G^{m}\right]
$$

An identification of $[H]=[W][G]$ with equation (10) gives:

$$
[W]=v_{p}\left[W^{p}\right]+v_{m}\left[W^{m}\right]
$$

As a result, the generalized stiffness matrix $[K]$ is obtained:

$$
[K]=[[Q][W]-[P]][[Q]-[P][W]]^{-1}
$$




\subsubsection{Extension of the matrix method to 1-3 piezocomposites}

By applying the relationship of continuity and adopting the methodology as presented in the case of the 2-2 connectivity, the effective electromechanical stiffness matrix is obtained in the case of the 13 connectivity.

\subsection{Homogenization models of Smith and Cha}

Actual properties piezocomposite which are most appropriate in the thickness vibration mode are given by the following equations, where all parameters are represented by the IEEE standard notation [13, 14]. According to Smith et al. [13], for a 1-3 connectivity piezocomposite, we get:

$$
\begin{aligned}
& c_{33}^{E, \text { eff }}=v_{p}\left(c_{33}^{E, p}-\frac{2 v_{m}\left(c_{13}^{p}-c_{12}^{m}\right)^{2}}{v_{p}\left(c_{11}^{m}+c_{12}^{m}\right)+v_{m}\left(c_{11}^{p}+c_{12}^{p}\right)}\right)+v_{m} c_{33}^{m} \\
& e_{33}^{e f f}=v_{p}\left(e_{33}^{p}-\frac{2 v_{m} e_{31}^{p}\left(c_{13}^{p}-c_{12}^{m}\right)}{v_{p}\left(c_{11}^{m}+c_{12}^{m}\right)+v_{m}\left(c_{11}^{p}+c_{12}^{p}\right)}\right) \\
& \varepsilon_{33}^{S, \text { eff }}=v_{p}\left(\varepsilon_{33}^{S, p}+\frac{2 v_{m}\left(e_{31}^{p}\right)^{2}}{v_{p}\left(c_{11}^{m}+c_{12}^{m}\right)+v_{m}\left(c_{11}^{p}+c_{12}^{p}\right)}\right)+v_{m} \varepsilon_{33}^{m}
\end{aligned}
$$

According to Cha et al. [14], for a 2-2 connectivity piezocomposite, we obtain:

$$
\begin{aligned}
& c_{33}^{E, \text { eff }}=v_{p}\left(c_{33}^{E, p}-\frac{v_{m}\left(c_{13}^{p}-c_{12}^{m}\right)^{2}}{v_{p} c_{11}^{m}+v_{m} c_{11}^{p}}\right)+v_{m} c_{33}^{m} \\
& e_{33}^{e f f}=v_{p}\left(e_{33}^{p}-\frac{v_{m} e_{31}^{p}\left(c_{13}^{p}-c_{12}^{m}\right)}{v_{p} c_{11}^{m}+v_{m} c_{11}^{p}}\right) \\
& \varepsilon_{33}^{S, \text { eff }}=v_{p}\left(\varepsilon_{33}^{S, p}+\frac{v_{m}\left(e_{31}^{p}\right)^{2}}{v_{p} c_{11}^{m}+v_{m} c_{11}^{p}}\right)+v_{m} \varepsilon_{33}^{m}
\end{aligned}
$$

The model of W.A. Smith [13] is valid when the lateral spatial scale of the composite is sufficiently fine that the composite can be treated as an effective homogeneous medium. In order to find the expressions of the elastic, piezoelectric and dielectric coefficients above, W.A. Smith [13] gave the relations of behavior following three directions $(x, y, z)$ for the matrix phase (exponent $m$ ) and the piezoelectric phase (exponent $p$ ). It is thus supposed that the strain along the $x$ direction is the same as that along the $y$ direction, both in the matrix phase and the piezoelectric phase $\left(S_{1}{ }^{m}=S_{2}{ }^{m}\right.$ and $S_{1}^{p}=S_{2}^{p}$ ), what makes it possible to find the following relations:

$$
\left\{\begin{array} { l } 
{ T _ { 1 } ^ { m } = ( c _ { 1 1 } + c _ { 1 2 } ) S _ { 1 } ^ { m } + c _ { 1 2 } S _ { 3 } ^ { m } } \\
{ T _ { 3 } ^ { m } = 2 c _ { 1 2 } S _ { 1 } ^ { m } + c _ { 1 1 } S _ { 3 } ^ { m } } \\
{ D _ { 3 } ^ { m } = \varepsilon _ { 3 3 } \cdot E _ { 3 } ^ { m } }
\end{array} \quad \text { and } \quad \left\{\begin{array}{l}
T_{1}^{p}=\left(c_{11}^{E}+c_{12}^{E}\right) S_{1}^{p}+c_{13}^{E} S_{3}^{p}-e_{31} E_{3}^{p} \\
T_{3}^{p}=2 c_{13}^{E} S_{1}^{p}+c_{33}^{E} S_{3}^{p}-e_{33} E_{3}^{p} \\
D_{3}^{p}=\varepsilon_{33}^{S} . E_{3}^{p}+e_{33} S_{3}^{p}+2 e_{31} S_{1}^{p}
\end{array}\right.\right.
$$

J.H. Cha [14] supposed that the length of the 2-2 piezocomposite in the $y$ direction can be considered infinite compared with that in the $x$ direction. The strain along the $y$ direction is the same both in the matrix phase and in the piezoelectric phase. It can be considered null $\left(S_{2}{ }^{m}=S_{2}{ }^{p}=0\right)$ and we obtain the following equations: 


$$
\left\{\begin{array} { l } 
{ T _ { 1 } ^ { m } = c _ { 1 1 } S _ { 1 } ^ { m } + c _ { 1 2 } S _ { 3 } ^ { m } } \\
{ T _ { 3 } ^ { m } = c _ { 1 2 } S _ { 1 } ^ { m } + c _ { 1 1 } S _ { 3 } ^ { m } } \\
{ D _ { 3 } ^ { m } = \varepsilon _ { 3 3 } \cdot E _ { 3 } ^ { m } }
\end{array} \quad \text { and } \quad \left\{\begin{array}{l}
T_{1}^{p}=c_{11}^{E} S_{1}^{p}+c_{13}^{E} S_{3}^{p}-e_{31} E_{3}^{p} \\
T_{3}^{p}=c_{13}^{E} S_{1}^{p}+c_{33}^{E} S_{3}^{p}-e_{33} E_{3}^{p} \\
D_{3}^{p}=\varepsilon_{33}^{s} \cdot E_{3}^{p}+e_{33} S_{3}^{p}+e_{31} S_{1}^{p}
\end{array}\right.\right.
$$

Ultimately, the difference between these expressions is that for the model of W.A. Smith [13], they are applicable only to the piezocomposites of 1-3 connectivities, whereas the expressions developed by J.H. Cha [14] can be adapted only to the 2-2 piezocomposites connectivities. Among the applications of such homogenization models, C.N. Della et al. [5] studied the performance of 1-3 piezocomposites with the active and passive phases using the Mori-Tanaka model [4]. The electromechanical parameters show that the use of an active polymer phase can improve the performance of the studied hydrophone. For transducer applications, the effective expressions of several key parameters are usually used, i.e. the acoustic impedance $Z_{L}^{e f f}$, longitudinal velocity $c_{L}^{e f f}$ and the electromechanical coupling factor $k_{t}^{\text {eff: }}$ :

$$
\begin{aligned}
& \left\{\begin{array}{l}
Z_{L}^{\text {eff }}=\sqrt{c_{33}^{D, \text { eff }} \rho^{\text {eff }}} \\
c_{L}^{\text {eff }}=\sqrt{\frac{c_{33}^{\text {,eff }}}{\rho^{\text {eff }}}} \\
k_{t}^{\text {eeff }}=\frac{e_{33}^{\text {eff }}}{\sqrt{c_{33}^{\text {,eff }} \varepsilon_{33}^{S, \text { eff }}}}
\end{array}\right. \\
& \text { with }\left\{\begin{array}{l}
\rho^{\text {eff }}=v_{p} \rho^{p}+\left(1-v_{p}\right) \rho^{m} \\
c_{33}^{D, \text { eff }}=c_{33}^{E, \text { eff }}+\frac{\left(e_{33}^{\text {eff }}\right)^{2}}{\varepsilon_{33}^{S, \text { eff }}}
\end{array}\right.
\end{aligned}
$$

\section{Homogenization}

For numerical calculations, we will use as an example a piezocomposite made of two phases: the first one being the polymer Araldite D, and the second one being the piezoelectric ceramic PZT-7A. The electromechanical characteristics of these materials are summarized in Table 1.

Table 1: Electromechanical properties of piezoelectric materials [15].

\begin{tabular}{ccccccccc}
\hline Material & $\begin{array}{c}c_{11}^{E} \\
(\mathrm{GPa})\end{array}$ & $\begin{array}{c}c_{12}^{E} \\
(\mathrm{GPa})\end{array}$ & $\begin{array}{c}c_{13}^{E} \\
(\mathrm{GPa})\end{array}$ & $\begin{array}{c}c_{33}^{E} \\
(\mathrm{GPa})\end{array}$ & $\begin{array}{c}e_{31} \\
\left(\mathrm{C} / \mathrm{m}^{2}\right)\end{array}$ & $\begin{array}{c}e_{33} \\
\left(\mathrm{C} / \mathrm{m}^{2}\right)\end{array}$ & $\begin{array}{c}\rho \\
\left(\mathrm{kg} / \mathrm{m}^{3}\right)\end{array}$ & $\frac{\varepsilon_{33}^{S}}{\varepsilon_{0}}$ \\
\hline Araldite D & 8.0 & 4.4 & 4.4 & 8.0 & 0 & 0 & 1150 & 4.2 \\
\hline PZT-7A & 148 & 76.2 & 74.2 & 131 & -2.1 & 9.5 & 7600 & 235 \\
\hline
\end{tabular}

The effective properties resulting from these homogenization models are plotted as a function of the volume fraction of PZT-7A. For a 1-3 connectivity, homogenization results of analytical results are compared with the experimental results, those extracted from the published work of H.L.W. Chan and J. Unsworth [15]. 


\subsection{Homogenization results for the $2-2$ connectivity}

The following Figure 3 illustrates the electromechanical characteristics (equation (14)) obtained as a function of the volume fraction $v_{f}$ of the PZT-7A piezoelectric phase.

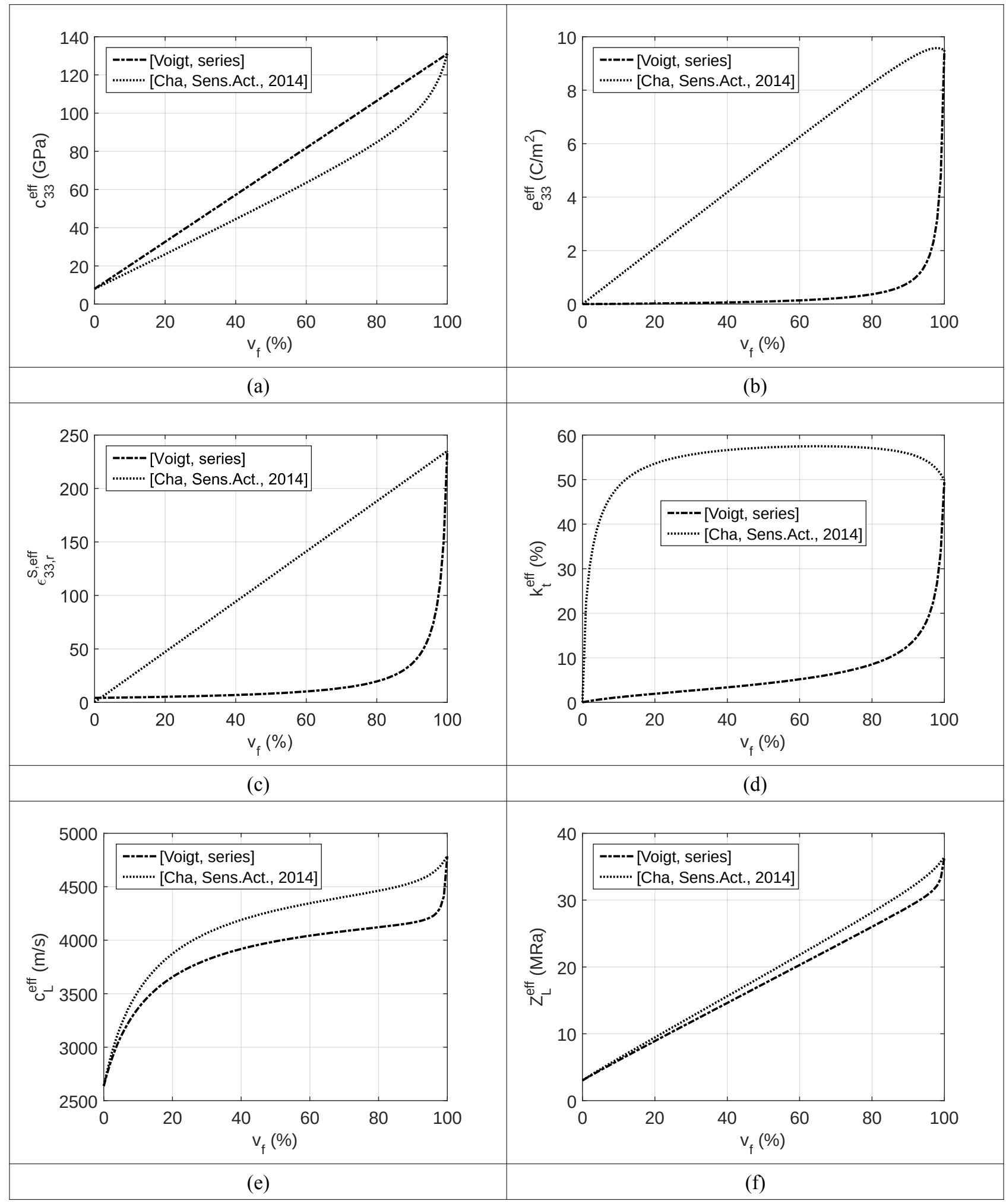

Figure 3: Homogenized 2-2 piezocomposite made of a PZT-7A/Araldite D as a function of the volume fraction $v_{f}$ of ceramic PZT-7A : (a) elastic constant $c_{33}^{\text {eff }}$, ,(b) piezoelectric constant $e_{33}^{\text {eff }}$, (c) relative dielectric constant $\varepsilon_{33}^{S, e f f} / \varepsilon_{0}$, , (d) electromechanical coupling coefficient $k_{t}^{\text {eff }}$, (e) longitudinal wave velocity $c_{L}^{\text {eff }}$ and (f) longitudinal acoustical impedance $Z_{L}^{\text {eff }}$. 


\subsection{Homogenization results for the 1-3 connectivity}

The following Figure 4 illustrates the homogenized electromechanical characteristics (equation (13)) obtained as a function of the volume fraction of the PZT-7A piezoelectric phase.

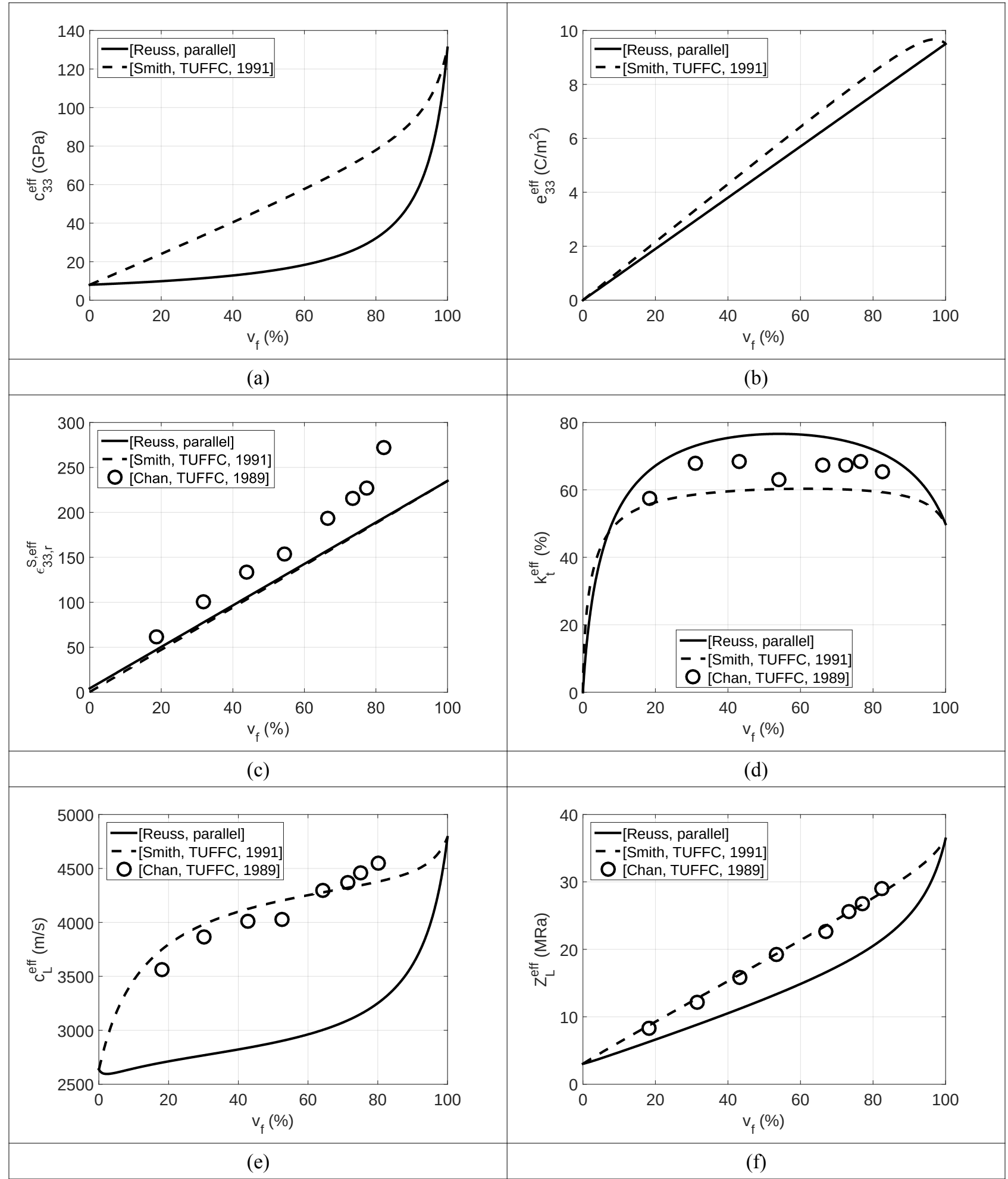

Figure 4: Homogenized 1-3 piezocomposite made of a PZT-7A/Araldite D as a function of the volume fraction $v_{f}$ of ceramic PZT-7A : (a) elastic constant $c_{33}^{\text {eff }}$, , (b) piezoelectric constant $e_{33}^{\text {eff }}$, (c) relative dielectric constant $\varepsilon_{33}^{S, \text { eff }} / \varepsilon_{0}$, (d) electromechanical coupling coefficient $k_{t}^{\text {eff }}$, (e) longitudinal wave velocity $C_{L}^{\text {eff }}$ and (f) longitudinal acoustical impedance $Z_{L}^{\text {eff }}$. Experimental data are extracted from refrence [15]. 


\subsection{Analysis and discussion}

In the previous section, various homogenization models were implemented to determine the effective characteristics of the piezocomposite material depending on the volume fraction of the PZT-7A piezoelectric phase. It can be noted that for the elastic coefficients in 2-2 connectivity, the upper bound is delimited by the Voigt model; the lower bound is that given by the Reuss model which gives the same formulation as that of the matrix model.

Meanwhile, the Cha model gives an intermediate result. Regarding the piezoelectric and dielectric coefficients, Voigt and matrix models are lower bounds. As a result, the electromechanical coupling coefficient combines these intermediate results and is directly related to the performance of the piezocomposite transducer. In terms of 2-2 connectivity, the electromechanical coefficient of coupling tends towards $60 \%$ for the Cha model.

In the case of the 1-3 connectivity, there is a coincidence between the elastic coefficients resulting from the matrix model and that of the Smith model. As expected, the Reuss model is the lower bound. For the piezoelectric coefficient of the 1-3 piezocomposite, a substantially linear variation is observed. In terms of the dielectric coefficient, Reuss, matrix and Smith models have a linear evolution and coincide. Finally, for the electromechanical coupling coefficient, Reuss model achieves a value that is above $75 \%$. In general, the values of the electromechanical coupling in the 1-3 connectivity are well above $50 \%$ for volume fractions between $10 \%$ and $100 \%$. This confirms the fact that the piezocomposite used for the manufacture of transducers are most often 1-3 connectivity.

After a comparative study of the models above, it appears that the analytically obtained results are close to experimental measurements. In the case of acoustic impedance, velocity longitudinal and electromechanical coupling factor, we note that Smith and matrix models are close to the experimental values from the model proposed by Reuss. In terms of the dielectric constant, the three models are linear and coincide. In general, we see that the homogenized piezocomposite characteristics vary depending on the model and piezocomposite connectivity used. The effective elastic, piezoelectric and dielectric coefficients are bounded by those of the matrix and those of the piezoelectric phase, and can lead to an improved electromechanical coupling factor. As a result, in the case of 1-3 connectivities, the effective thickness coupling factor $k_{t}^{e f f}$ ( $<50 \%$ for most of the ceramics) tends towards the $k_{33}$ electromechanical coupling factor ( $>70 \%$ for optimized volume fractions). Finally, we note that for piezocomposite 2-2 and 1-3 connectivities, the various homogenization models are ranging in the limits given by Voigt and Reuss. 


\section{Transducer application}

On the basis of the simulation results of the piezocomposite materials, the electroacoustic response of transducers having some of those properties are simulated using the KLM model [16]. In the limit of the acoustical impedance of the piezoelectric material, the choice of the backing material is of first importance. When this critical value is exceeded, the drawbacks are canceling the benefits. The higher the acoustical impedance of the backing, the higher the damping of the piezoelectric resonance, i.e. amplitude loss and low frequency shift. A trade-off between sensitivity (amplitude) and echo duration (time response duration at $-6 \mathrm{~dB}$ ) can be found on the basis of a performance index designed by the end-user applications [17]. Therefore, a given transducer configuration based on a PZT-7A piezoelectric material radiating in water is studied. The specifications were drawn up with a view to an application in medical imaging. The specific requirements are a thickness mode resonance frequency at $f_{0}=12 \mathrm{MHz}$, an active surface at $S=50 \mathrm{~mm}^{2}$, a front medium which is water with $Z_{f}=1.5 \mathrm{MRa}$, and an electrical environment fixed at $Z_{g}=Z_{r}=50 \Omega$. First, the impulse transfer function spectrum (Figure 5 (a)) and electroacoustic response (Figure 5 (b)) are simulated as a function of the acoustic impedance of the backing.

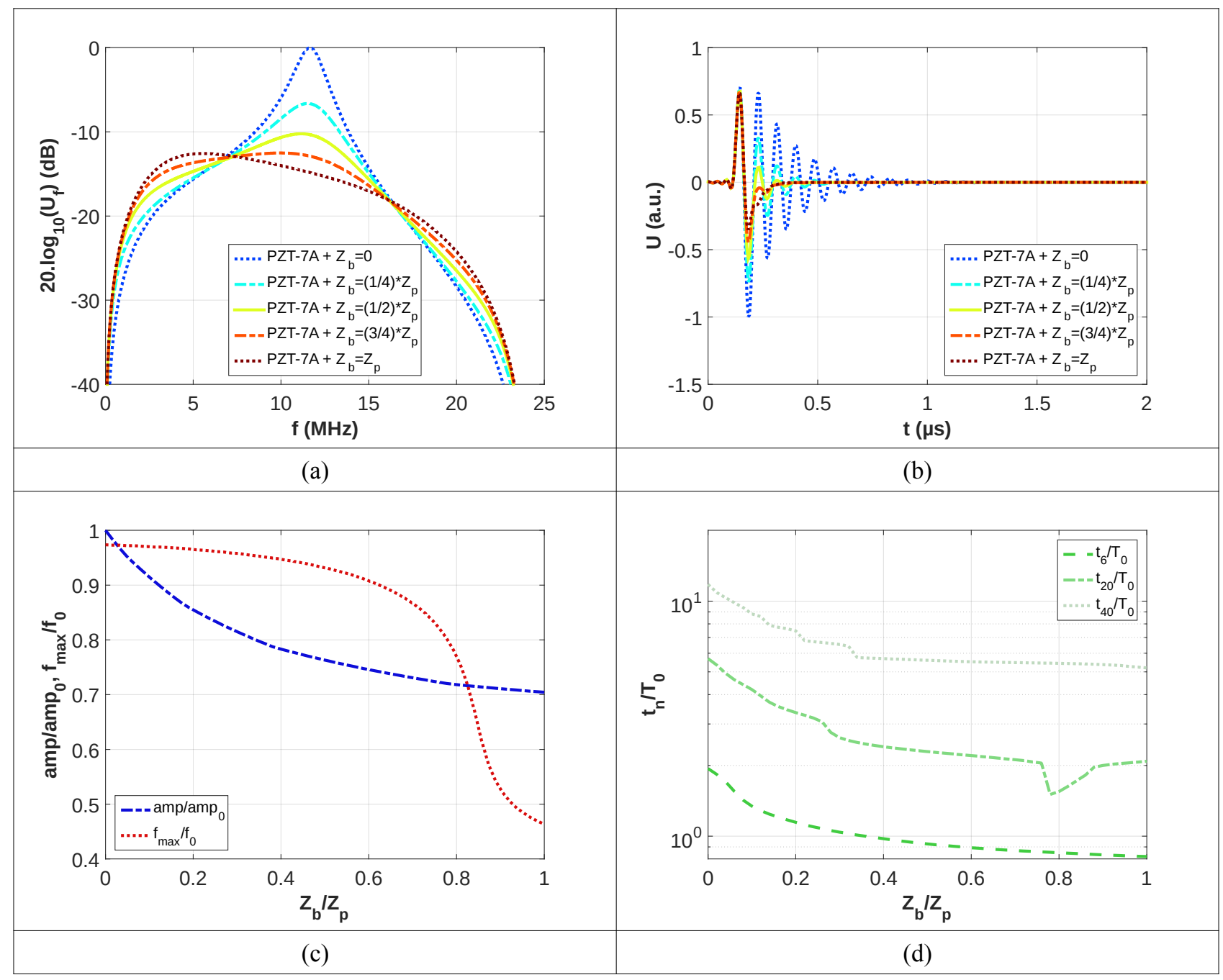




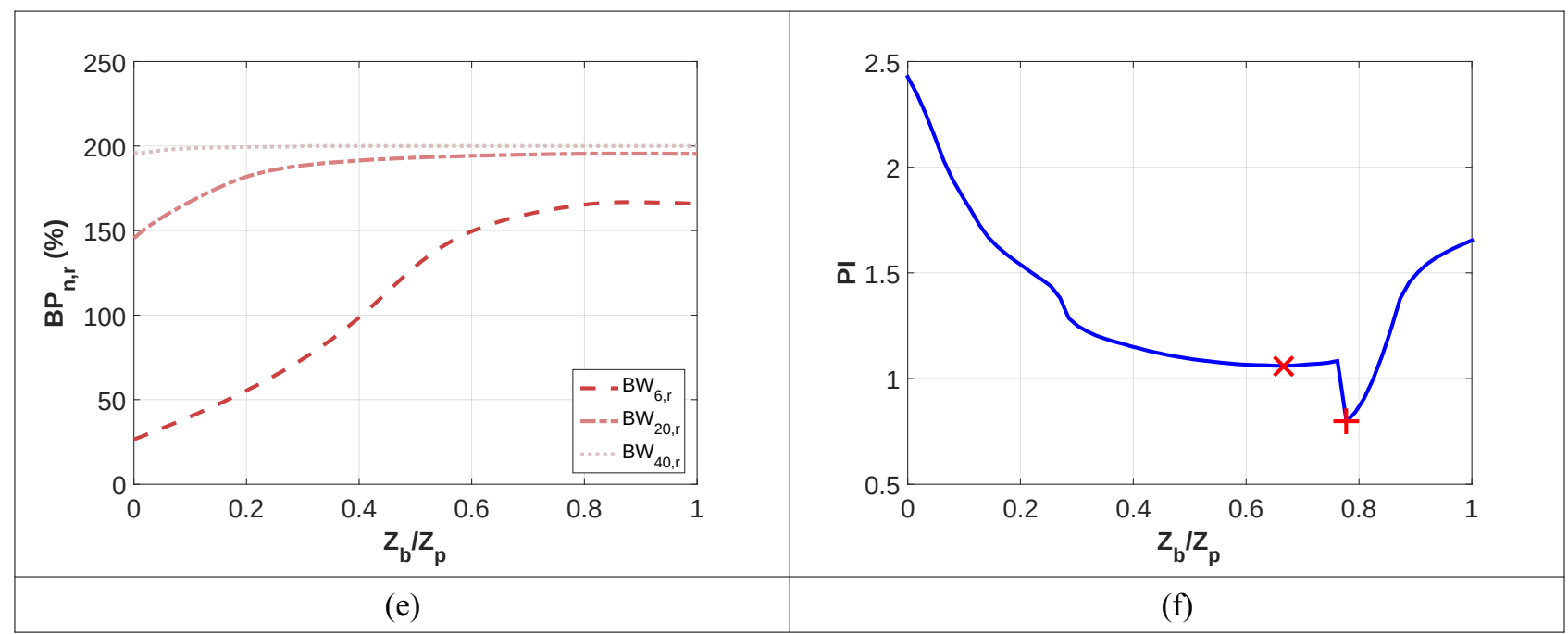

Figure 5: Comparison of immersion of PZT-7A based piezocomposites transducers with a loaded backing from $Z_{b}=0$ to $Z_{p}$ in terms of (a) transfer functions, (b) electroacoustic responses. Associated end-user elctroacoustic performances: (c) normalized amplitude and center frequency, (d) normalized duration $t_{n, r}=t_{n} / T_{0}$ at $-6,-20$ and $-40 \mathrm{~dB}$, (e) relative bandwidths $B W_{n, r}=B W_{n} / f_{c, n}$ at $-6,-20$ and $-40 \mathrm{~dB}$, (f) performance index $P I$ as defined in equation (19), as a function of the backing load $Z_{b} / Z_{p}$ from 0 to 1 .

The evolution of their properties is then evaluated and compared: the amplitude and center frequency are plotted in Figure $5(\mathrm{c})$, the normalized duration $t_{n, r}=t_{n} / T_{0}$ at $-6,-20$ and $-40 \mathrm{~dB}$ are in Figure $5(\mathrm{~d})$, the normalized bandwidths $B W_{n, r}=B W_{n} / f_{c, n}$ at $-6,-20$ and $-40 \mathrm{~dB}$ are in Figure 5 (e). On the basis of these estimators, the performance of the parameters may be summarized in a global performance index.

Here, we used a performance index PI (equation (19)) designed for ultrasound imaging, including $t_{6, r}$ the normalized time response duration at $-6 \mathrm{~dB}, t_{20, r}$ the normalized time response duration at $-20 \mathrm{~dB}, \operatorname{amp}_{n}$ the normalized maximum amplitude of the impulse response $U(t)$ and $f_{\max , n}$ the normalized frequency of the maximum of the transfer function $U_{f}(f)$ :

$$
P I=\log \left(\frac{t_{6, n} \cdot t_{20, n}}{a m p_{n} \cdot f_{\text {max }, n}}\right)
$$

The evolution of the performance index $P I$ for the studied configuration is illustrated in Figure 5 (f) and show two local minimums, for the normalized backing impedance at $Z_{b} / Z_{p}=2 / 3$ and 3/4, i.e. optimal performance for the application field which is ultrasound imaging. The first optimum of the performance index $P I$ around $Z_{b} / Z_{p}=2 / 3$ seems to correspond to the tendency of its evolution ("+" red cross in Figure 5 (f)), whereas the second one correspond to a local breakdown at $Z_{b} / Z_{p}=3 / 4$ (" $\times$ " red cross in Figure 5 (f)). This second optimum value is due to the $t_{20, r}$ parameter (Figure 5 (d)) which shows a similar decrease in this area. This can be explained by the arch of the impulse response that do not cross the threshold. Nevertheless, this local minimum may be disturbed by imperfections of a real transducer, and should not be taken into account. 
On the basis of the simulation results discussed in the previous parts, two optimal configuration have been selected: $v_{f}=60 \%$ for the piezocomposite $2-2$ connectivity and $v_{f}=50 \%$ for the piezocomposite 1-3 connectivity. These configurations were studied in a similar electroacoustic environment, i.e. water in the front face $Z_{f}=1.5 \mathrm{MRa}$, coupled in the back face with a backing having an acoustic impedance $Z_{b} / Z_{p}=2 / 3$, and a standard $50 \Omega$ electrical environment. Both of the optimal piezocomposite configurations exhibit a higher sensitivity and widen bandwidth but lower resonance frequency, i.e. slightly reduced sensitivity and electroacoustic response duration, which are constituting a good trade-off for acoustical imaging. This result can be confirmed by the improved transfer function spectrum (Figure 6 (a)) and electroacoustic response (Figure 6 (b)) for the two piezocomposite configurations when compared to a pure PZT-7A piezoelectric active element.

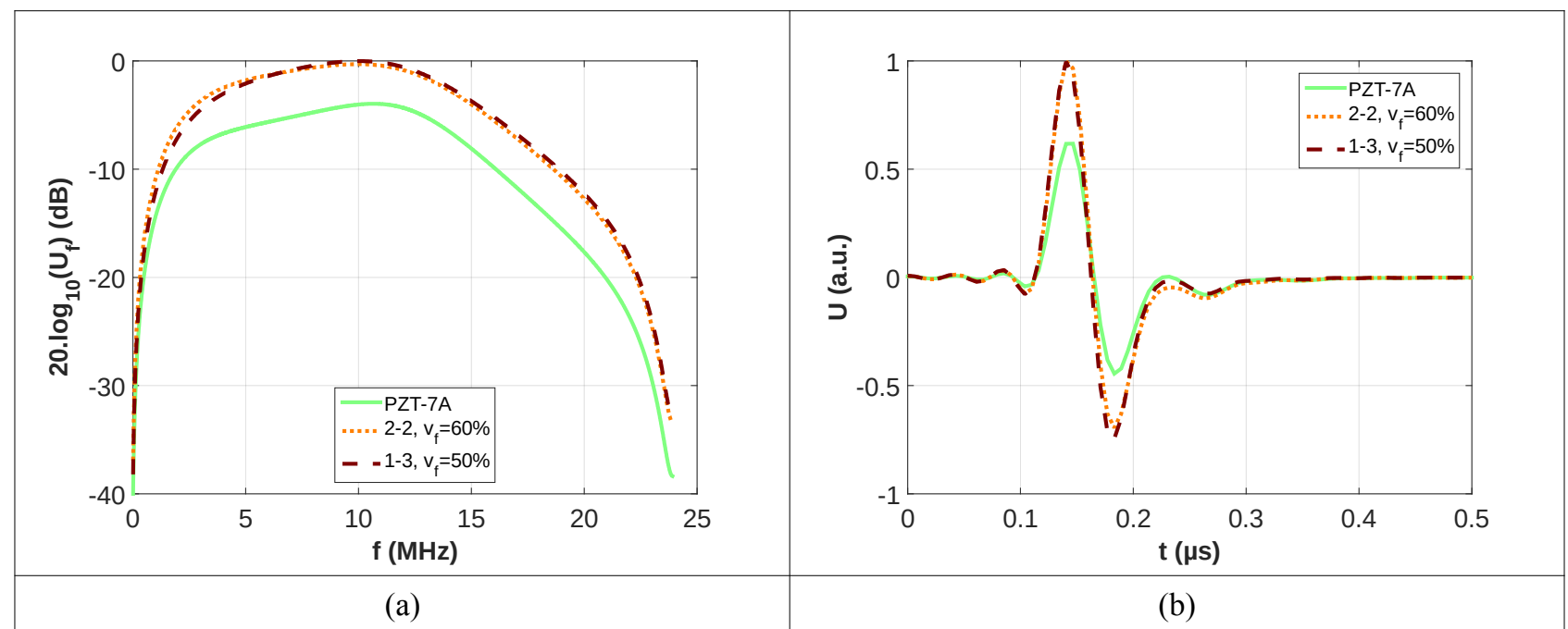

Figure 6: Comparison of immersion transducers with and epoxy backing in terms of (a) transfer functions and (b) electroacoustic responses of piezocomposites based on PZT-7A.

The obtained transfer function spectrum and electroacoustic resonance can be characterized in order to emphasize the interest of such piezocomposite compositions. A trade-off between some of these parameters can be compared in terms of performance for acoustical imaging applications. As defined by the performance index (equation (19)), it is evaluated at $P I=1.05,0.27$ and 0.27 for the three studied piezoelectric configurations: PZT-7A, 2-2 PZT-7A/Araldite D piezocomposite with $v_{f}=60 \%, 1-3$ PZT-7A/Araldite D piezocomposite with $v_{f}=50 \%$, respectively.

\section{Conclusion}

In this work, a comparative study was lead between several homogenization models applied to piezocomposite of 2-2 and 1-3 connectivities. It appears that for the acoustic impedance, velocity longitudinal and the electromechanical coupling factor, the Smith and matrix models are close to the 
experimental values. In terms of dielectric constant, the three models are linear and coincide. These homogenization results for piezocomposite characteristics strongly depend on the considered connectivity as well as on the constitutive parts. These homogenization models lead to determine the optimal volume fraction of piezoelectric phase according to a chosen criterion. Finally, an optimal configuration can be designed following an end-user application. As a result, a performance index dedicated to ultrasound imaging application is presented as a good trade-off between sensitivity and resolution. This performance index is then used and discussed to demonstrate an optimal design for high resolution and high sensitivity piezocomposite transducer.

\section{Data availability}

The raw/processed data required to reproduce these findings cannot be shared at this time due to technical or time limitations.

\section{References}

[1] Pham Thi M., Hladky-Hennion A.C., Le Khanh H., Tran-Huu-Hue L.P., Lethiecq M., Levassort F., "Large area 0-3 and 1-3 piezoelectric composites based on single crystal PMN-PT for transducer applications", Physics Procedia, Vol.3, p.897-904, 2010.

[2] Zhou D., Lam K.H., Chen Y., Zhang Q., Chiu Y.C., Luo H., Dai J., Chan H.L.W., "Lead-free piezoelectric single crystal based 1-3 composites for ultrasonic transducer applications", Sensors and Actuators A: Physical, Vol.182, p.95-100, 2012.

[3] Ritter T.A., Shrout T.R., Tutwiler R., and Shung K.K., "A 30-MHz Piezo-Composite Ultrasound Array for Medical Imaging Applications", IEEE Transactions on Ultrasonics, Ferroelectrics, and Frequency Control, Vol.49(2), p.217-230, 2002.

[4] Levin V.M., Sabina F.J., Bravo-Castillero J., Guinovart-Díaz R., Rodríguez-Ramos R., Valdiviezo-Mijangos O.C., "Analysis of effective properties of electroelastic composites using the self-consistent and asymptotic homogenization methods", International Journal of Engineering Science, Vol.46 (8), p.818-834, 2008.

[5] Della C.N., Shu D., "On the performance of 1-3 piezoelectric composites with a passive and active matrix", Sensors and Actuators A: Physical, Vol.140, p.200-206, 2007.

[6] Wang C., Liu Y., Zhang R., Cao W., "Effect of kerf filler on the electromechanical coupling coefficient of 1-3 piezoelectric composites", Journal of Alloys and Compounds, Vol.651, p.643-647, 2015.

[7] Newnham R.E., Bowen L.J., Klicker K.A., Cross L.E., "Composite piezoelectric transducers", Materials in engineering, Vol.2, p.93-106, 1980. 
[8] L.D. Landau and E.M. Lifshitz, "Theory of Elasticity", Course of Theoretical Physics, Ed. Butterworth-Heinemann, Vol.7, Third Edition, 1986.

[9] Voigt W., "Lehzbook der Kristallphysik", 962 p., Teuber, Leipzig, Second edition, 1928.

[10] Reuss J., "Berechnung des fleissgrenze von mischkristallen auf grud der platizitatsbedingung für einkristalle: Z. Angrew Math.Mech.", Vol.9 (1), p.49-58, 1929.

[11] Hashimoto K.Y., Yamaguchi M., "Elastic, piezoelectric and dielectric properties of composite materials", Proc. IEEE Ultrason. Symp., p.697-702, 1986.

[12] Levassort F., Lethiecq M., Certon D., Patat F., "A matrix method for modeling electroelastic moduli of 0-3 piezo-composites", IEEE Transactions on Ultrasonics, Ferroelectrics, and Frequency Control, Vol.44 (2), p.445-452, 1997.

[13] Smith W.A., Auld B.A., "Modeling 1-3 composite piezoelectrics: thickness-mode oscillations", IEEE Transactions on Ultrasonics, Ferroelectrics, and Frequency Control, Vol.38 (1), p.40-47, 1991. [14] Cha J.H., Chang J.H., "Development of 15 MHz 2-2 piezocomposite ultrasound linear array transducers for ophthalmic imaging", Sensors and Actuators A, Vol.217, p.39-48, 2014.

[15] Chan H.L.W., Unsworth J., "Simple model for piezoelectric ceramic/polymer 1-3 composites used in ultrasonic transducer applications", IEEE Transactions on Ultrasonics, Ferroelectrics, and Frequency Control, Vol.36 (4), p.434-441 1989.

[16] Maréchal P., Levassort F., Tran-Huu-Hue L.P., Lethiecq M., "Lens-focused transducer modeling using an extended KLM model", Ultrasonics, Vol.46, p.155-167, 2007.

[17] Maréchal P., Levassort F., Tran-Huu-Hue L.P., Lethiecq M., "Electro-acoustic response at the focal point of a focused transducer as a function of the acoustical properties of the lens", Proceeding of the 5th World Congress on Ultrasonics, 2003. 